\title{
Comportamento ingestivo de cordeiros e digestibilidade dos nutrientes de dietas contendo alta proporção de concentrado e diferentes fontes de fibra em detergente neutro
}

\section{Clayton Quirino Mendes ${ }^{1}$, Vicente de França Turino ${ }^{1}$, Ivanete Susin², Alexandre Vaz Pires², Janice Barreto de Morais $^{1}$, Renato Shinkai Gentil ${ }^{1}$}

\footnotetext{
${ }^{1}$ Programa de pós-graduação em Ciência Animal e Pastagens - ESALQ/USP - Piracicaba, SP.

2 Departamento de Zootecnia - ESALQ/USP - Piracicaba, SP. Pesquisador do CNPq.
}

RESUMO - Dois experimentos foram realizados para avaliar os efeitos da substituição da fibra em detergente neutro (FDN) do bagaço de cana-de-açúcar in natura pela FDN da casca de soja em dietas com alta proporção de concentrado sobre o comportamento ingestivo de cordeiros e a digestibilidade dos nutrientes. No primeiro experimento, 60 cordeiros com 16,4 \pm 0,3 $\mathrm{kg}$ de peso corporal e idade inicial de $67 \pm 2$ dias foram distribuídos em delineamento de blocos completos casualizados, em esquema fatorial $2 \times 2+1$, composto de duas fontes de FDN (bagaço de cana in natura ou casca de soja), dois teores de FDN (14 ou 18\%) e uma dieta controle contendo $100 \%$ de concentrado. No segundo experimento, cinco cordeiros foram distribuídos em quadrado latino $5 \times 5$ e submetidos às mesmas dietas do experimento 1 . As dietas contendo as fontes de fibra promoveram maior consumo de matéria seca, matéria orgânica e FDN e maior tempo de ingestão, ruminação e mastigação (minutos/dia) em comparação à dieta contendo 100\% de concentrado. As dietas contendo o bagaço de canade-açúcar in natura proporcionaram maior atividade de ruminação que aquelas com casca de soja. Entretanto, a utilização da casca de soja proporcionou menor atividade de mastigação e maior digestibilidade dos nutrientes em relação ao bagaço de cana-de-açúcar in natura. A casca de soja pode ser utilizada como única fonte adicional de FDN em dietas contendo alto teor de concentrado para cordeiros.

Palavras-chave: bagaço de cana-de-açúcar, casca de soja, fibra efetiva, ruminação, subproduto

\section{Lamb feeding behavior and nutrient digestibility of high concentrate diets with different neutral detergent fiber sources}

\begin{abstract}
Two experiments were performed to determine the effects of replacing the neutral detergent fiber from sugarcane bagasse with soybean hulls neutral detergent fiber in high concentrate diets on lamb feeding behavior and apparent nutrient digestibility. In the first experiment, 60 ram lambs with $16.4 \pm 0.3 \mathrm{~kg}$ body weight and $67 \pm 2$ days old were allotted to a complete randomized block design as a $2 \times 2+1$ factorial arrangement of treatments, consisting of two neutral detergent fiber sources (sugar cane bagasse or soybean hulls), two neutral detergent fiber levels (14 or 18\%) and a control diet containing $100 \%$ concentrate. In the second experiment, five ram lambs were assigned to a $5 \times 5$ Latin square design and fed the same experimental diets as experiment one. Diets containing fiber sources increased dry matter, organic matter and neutral detergent fiber intakes and promoted longer feeding, ruminating and chewing times (minutes/day) compared to the diet containing $100 \%$ concentrate. The diets containing sugarcane bagasse increased rumination activity compared to those with soybean hulls as fiber source. However, diets containing soybean hulls showed lower chewing activity and greater nutrient digestibility compared to the sugarcane bagasse diets. Soybean hulls can be used as the sole source of additional neutral detergent fiber for lambs fed high concentrate diets.
\end{abstract}

Key Words: byproduct, effective fiber, rumination, soybean hulls, sugarcane bagasse

\section{Introdução}

A casca de soja é um subproduto da indústria de processamento da soja e é considerada como suplemento energético (NRC, 1985), pois seu fornecimento aos ruminantes permite obter desempenhos comparáveis aos obtidos com do milho, devido à boa digestibilidade da parede celular, basicamente composta de celulose (Anderson et al., 1988). Alguns autores consideram a casca do grão de soja como ingrediente volumoso e concentrado, por possuir fibra efetiva e, dentro de certos limites, funcionar como grão de cereal em termos de disponibilidade de energia (Sarwar et al., 1991).

Recebido em 13/5/2008 e aprovado em 16/3/2009.

Correspondências devem ser enviadas para: ivasusin@esalq.usp.br. 
O bagaço de cana-de-açúcar in natura (BIN), subproduto resultante da moagem de cana-de-açúcar, encontrado com facilidade nas usinas, tem sido utilizado com frequência como fonte de fibra na alimentação de ruminantes de grande porte. Segundo Henrique et al. (2007), esse subproduto é um alimento rico em constituintes da parede celular, contém baixo conteúdo celular e possui baixa digestibilidade e baixa densidade.

Em dietas com alta proporção de ingredientes concentrados, é mais seguro o uso de um teor mínimo de fibra, capaz de estimular a mastigação e permitir ambiente ruminal adequado para não prejudicar o desempenho animal. Alguns trabalhos indicam que o uso de pequena quantidade de bagaço de cana-de-açúcar in natura como fonte de fibra em dietas para bovinos de corte pode evitar problemas metabólicos, sem prejudicar o desempenho, quando utilizada alta proporção de concentrado (Bulle et al., 2002).

A casca de soja é pouco efetiva para estimular a ruminação, pois possui tamanho reduzido de partículas. Entretanto, o seu alto teor de fibra digestível (74\% de FDN), 46\% composta de celulose e 18\% de hemicelulose (Anderson et al., 1988), promove padrão de fermentação semelhante ao de forragem, o que contribui para manutenção do pH ruminal e não prejudica o desempenho animal e a digestibilidade dos nutrientes. Existem poucas informações sobre o uso de casca de soja e/ou bagaço de cana in natura na alimentação de cordeiros. Além disso, existem poucos resultados referentes ao teor de FDN em dietas com alta proporção de concentrado para ovinos.

O objetivo neste trabalho foi avaliar o uso de dietas contendo dois níveis de casca de soja em substituição à fibra em detergente neutro do bagaço de cana-de-açúcar in natura sobre o comportamento ingestivo, o consumo e a digestibilidade da matéria seca, matéria orgânica e da FDN no trato digestório total de ovinos.

\section{Material e Métodos}

No primeiro experimento, avaliou-se o comportamento ingestivo dos animais utilizando-se 60 cordeiros da raça Santa Inês, com 16,4 \pm 0,3 kg de peso corporal e idade inicial de $67 \pm 2$ dias. Os animais foram alojados em baias com piso de concreto, com dimensões de 4,00 × 1,35 m, cocho coberto e bebedouro, sendo dois animais por baia e seis baias por tratamento. Os cordeiros foram vacinados contra clostridioses e everminados (mediante prévio exame parasitológico de fezes) antes de iniciar o período experimental.
O período experimental teve duração de 84 dias, divididos em três subperíodos de 28 dias. No 26으 dia de cada subperíodo experimental, foi avaliado o comportamento ingestivo de todos os animais, individualmente, durante 24 horas, com observações a cada cinco minutos (Weidner \& Grant, 1994), para determinação do tempo gasto com ingestão e ruminação em minutos/dia. O tempo total despendido em cada atividade foi calculado multiplicando-se o número total de observações por cinco. A atividade de mastigação foi calculada pelo somatório das atividades de ingestão e ruminação, conforme Armentano \& Pereira (1997). As atividades de ruminação, ingestão e mastigação foram expressas em minutos/dia e minutos/g de FDN consumida.

Os tratamentos constaram de cinco dietas isonitrogenadas (Tabela 1): 100C = dieta composta por $100 \%$ de concentrado; BIN14 = dieta contendo 14\% FDN e composta de $95 \%$ de concentrado e $5 \%$ de BIN; BIN18 = dieta contendo 18\% FDN e composta de $90 \%$ de concentrado e $10 \%$ de BIN; CS14 = dieta contendo 14\% FDN e composta de $92 \%$ de concentrado e $8 \%$ de casca de soja (CS) e CS18 = dieta contendo 18\% FDN e composta de $88 \%$ de concentrado e $12 \%$ de casca de soja (Turino et al., 2007).

O delineamento experimental utilizado foi o de blocos casualizados, em arranjo fatorial $2 \times 2$ mais um tratamento controle. Cada baia foi considerada uma unidade experimental, totalizando cinco tratamentos e seis repetições. Os blocos foram definidos de acordo com o peso e a idade dos animais no início do experimento.

Os dados foram analisados pelo procedimento GLM do programa estatístico SAS (1999). As variáveis determinadas foram: tempos de ingestão, ruminação e mastigação em minutos/dia e minutos/g de FDN ingerida. Contrastes ortogonais foram utilizados para determinação dos efeitos principais: fonte (BIN $\times$ CS), teor de FDN (14\% $\times 18 \%$ ), interação teor $\times$ fonte e interação $100 \mathrm{C} \times$ outros (dieta $100 \%$ de concentrado $\times$ as outras quatro dietas) sobre as variáveis analisadas.

No segundo experimento, avaliaram-se o consumo e a digestibilidade dos nutrientes no trato digestório total. Cinco cordeiros da raça Santa Inês, com 35,6 kg de peso corporal e 5,5 meses de idade, foram distribuídos em delineamento experimental em quadrado latino $5 \times 5$. Os animais foram alojados em gaiolas metálicas individuais para ensaios de metabolismo, com dimensões de 1,3 × 0,55 m, providas de cocho e bebedouro.

As dietas experimentais foram as mesmas utilizadas no experimento 1 (Tabela 1). O período experimental teve duração total de 70 dias, divididos em cinco subperíodos de 
Tabela 1 - Ingredientes e composição nutricional das dietas experimentais (\%MS)

\begin{tabular}{|c|c|c|c|c|c|}
\hline \multirow[t]{3}{*}{ Item } & \multicolumn{5}{|c|}{ Dieta } \\
\hline & \multirow[t]{2}{*}{$100 C^{1}$} & \multicolumn{2}{|c|}{ Bagaço de cana-de-açúcar in natura ${ }^{2}$} & \multicolumn{2}{|c|}{ Casca de soja ${ }^{3}$} \\
\hline & & FDN14 & FDN18 & FDN14 & FDN18 \\
\hline \multicolumn{6}{|l|}{ Ingrediente } \\
\hline Milho moído grosso & 81,2 & 75,5 & 69,8 & 74,1 & 69,4 \\
\hline Farelo de soja & 15,3 & 16,0 & 16,7 & 14,6 & 15,2 \\
\hline Casca de soja & - & - & - & 7,8 & 11,9 \\
\hline Bagaço de cana-de-açúcar & - & 5,0 & 10,0 & - & - \\
\hline Suplemento mineral ${ }^{4}$ & 1,5 & 1,5 & 1,5 & 1,5 & 1,5 \\
\hline Calcário calcítico & 1,5 & 1,5 & 1,5 & 1,5 & 1,5 \\
\hline Cloreto de amônio & 0,5 & 0,5 & 0,5 & 0,5 & 0,5 \\
\hline \multicolumn{6}{|l|}{ Composição nutricional } \\
\hline Matéria seca & 89,3 & 87,2 & 85,9 & 89,9 & 89,4 \\
\hline Proteína bruta & 16,8 & 16,6 & 16,3 & 16,5 & 16,1 \\
\hline Fibra em detergente neutro & 11,1 & 14,6 & 18,1 & 14,5 & 19,0 \\
\hline \multicolumn{6}{|l|}{ Tamanho de partículas (\%) } \\
\hline$>4 \mathrm{~mm}$ & 10,1 & 8,6 & 16,4 & 6,6 & 7,3 \\
\hline $2-4 \mathrm{~mm}$ & 30,9 & 34,5 & 26,4 & 31,3 & 27,3 \\
\hline $2-1,18 \mathrm{~mm}$ & 21,5 & 20,4 & 18,7 & 19,8 & 19,2 \\
\hline$<1,18 \mathrm{~mm}$ & 37,5 & 36,5 & 38,5 & 42,3 & 46,2 \\
\hline
\end{tabular}

${ }^{1}$ Dieta contendo $100 \%$ de concentrado (sem fonte adicional de FDN).

2 Dietas contendo 14 ou $18 \%$ de FDN com bagaço de cana-de-açúcar in natura como fonte de FDN.

${ }^{3}$ Dietas contendo 14 ou $18 \%$ de FDN com casca de soja como fonte de FDN.

${ }^{4}$ Composição: P - 7,5\%; Ca - 24,7\%; Cl - 21.8\%; Na - 14,5\%; Mg - 1\%; S - 7\%; Mn - 1.100 ppm; Fe - 500 ppm; Zn - 4.600 ppm; Cu - 300 ppm; Co - 40 ppm; I - 55 ppm; Se - 30 ppm.

14 dias (dez para adaptação dos animais às dietas e quatro dias de colheita).

Os animais foram alimentados uma vez ao dia e os dados de consumo diário de MS por animal foram obtidos pela diferença entre a quantidade de alimento fornecido e

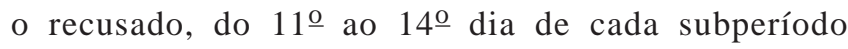
experimental. Durante os quatro dias de colheita, amostras das fezes e dos alimentos oferecidos e recusados foram colhidas e compostas por animal e sub-período, sendo conservadas a $-18^{\circ} \mathrm{C}$. Posteriormente, todas as amostras foram descongeladas e secas em estufas de ventilação forçada $\left(55-60^{\circ} \mathrm{C}\right)$, por 72 horas, de acordo com Goering \& Van Soest (1970). As amostras foram trituradas em moinho tipo Wiley com peneiras com crivos de $1 \mathrm{~mm}$ e analisadas para determinação das concentrações de matéria mineral e matéria seca (AOAC, 1990) e FDN de acordo com Van Soest et al. (1991). A digestibilidade aparente dos nutrientes foi calculada a partir da seguinte fórmula:

$$
\operatorname{DAN}(\%)=\frac{(\mathrm{MSC} \times \mathrm{NMS})-(\mathrm{MSF} \times \mathrm{NMF}) \times 100}{(\mathrm{MSC} \times \mathrm{NMS})}
$$

em que: DAN (\%) = digestibilidade aparente do nutriente; MSC = matéria seca consumida; MSF = matéria seca fecal; $\mathrm{NMF}=$ porcentagem do nutriente na matéria seca fecal; NMS = porcentagem do nutriente na matéria seca consumida.

Os dados foram analisados pelo procedimento GLM do programa estatístico SAS (1999). Uma vez detectadas respostas significativas, foram utilizados contrastes ortogonais como no primeiro experimento.

\section{Resultados e Discussão}

Todas as dietas que continham fonte adicional de fibra proporcionaram maior tempo de ingestão em minutos/ dia $(\mathrm{P}<0,05)$ em relação à dieta contendo $100 \%$ de concentrado. Os resultados de ingestão em minutos/dia indicam interação entre os teores e as fontes de FDN $(\mathrm{P}<0,05)$, demonstrando que a casca de soja promoveu maior tempo de ingestão quando a dieta continha $14 \%$ de FDN, enquanto o bagaço de cana in natura promoveu maior tempo de ingestão quando utilizados $18 \%$ de FDN (Tabela 2). O maior tempo de ingestão dos animais que receberam a dieta contendo $18 \%$ de FDN advinda do bagaço de cana-de-açúcar está relacionado ao tempo gasto pelos animais em selecionar os alimentos ingeridos, uma vez que o consumo foi de 1,05 g de FDN/minuto, enquanto, para a dieta composta por $18 \%$ de FDN proveniente da casca de soja, a ingestão foi de 1,74 g de FDN/minuto. Assim como neste estudo, Araujo et al. (2008a) avaliaram os efeitos da substituição do feno de "coastcross" pela casca de soja sobre o comportamento ingestivo de ovelhas em lactação e observaram redução linear no tempo gasto com a atividade de ingestão à medida que a casca de soja foi incluída na dieta e aumento na quantidade de MS ingerida com a inclusão da casca de soja.

O tempo de ruminação foi influenciado pelas fontes de FDN $(\mathrm{P}<0,05)$, uma vez que as dietas que continham bagaço de cana promoveram maior tempo de ruminação em relação àquelas contendo casca de soja como fonte 
Tabela 2 - Comportamento ingestivo dos cordeiros alimentados com as dietas experimentais

\begin{tabular}{|c|c|c|c|c|c|c|c|c|c|c|}
\hline \multirow[t]{3}{*}{ Item } & \multicolumn{10}{|c|}{ Dieta } \\
\hline & \multirow[t]{2}{*}{$100 \mathrm{C}^{1}$} & \multicolumn{2}{|c|}{$\begin{array}{c}\text { Bagaço de cana-de-açúcar } \\
\text { in natura }{ }^{2}\end{array}$} & \multicolumn{2}{|c|}{ Casca de soja ${ }^{3}$} & \multirow[t]{2}{*}{$\mathrm{EPM}^{4}$} & \multicolumn{4}{|c|}{ Efeito principal ${ }^{5}$} \\
\hline & & FDN14 & FDN18 & FDN14 & FDN18 & & Fonte & Teor & Teor $\times$ Fonte & $100 \mathrm{C} \times$ Outros \\
\hline \multicolumn{11}{|l|}{ Ingestão } \\
\hline Minutos/dia & 122 & 142 & 215 & 161 & 134 & 12,91 & 0,03 & NS & $<0,01$ & 0,01 \\
\hline $\begin{array}{c}\text { Minutos/g Fibra em } \\
\text { detergente neutro }\end{array}$ & 0,95 & 0,79 & 1,08 & 0,97 & 0,57 & 0,09 & NS & NS & $<0,01$ & NS \\
\hline \multicolumn{11}{|l|}{ Ruminação } \\
\hline Minutos/dia & 177 & 300 & 289 & 157 & 154 & 14,73 & $<0,01$ & NS & NS & 0,008 \\
\hline $\begin{array}{l}\text { Minutos/g Fibra em } \\
\text { detergente neutro }\end{array}$ & 1,38 & 1,68 & 1,43 & 0,93 & 0,67 & 0,08 & $<0,01$ & 0,004 & NS & 0,032 \\
\hline \multicolumn{11}{|l|}{ Mastigação } \\
\hline Minutos/dia & 299 & 442 & 504 & 318 & 288 & 19,50 & $<0,01$ & NS & 0,03 & 0,0001 \\
\hline
\end{tabular}

de fibra. Esses resultados estão de acordo com informações de Ipharraguerre \& Clark (2003) de que a substituição de forragem pela casca de soja na alimentação de vacas em lactação reduz as atividades de ingestão e ruminação.

Os resultados observados para as dietas que continham bagaço de cana-de-açúcar estão de acordo com os obtidos por Rabelo et al. (2008), que alimentaram bovinos de corte com $50 \%$ de bagaço de cana-de-açúcar - $45 \%$ de bagaço tratado sob pressão e vapor (BTPV) e 5\% de bagaço de cana in natura - como fonte de fibra e observaram ruminação média de 289 minutos/dia.

O tempo de ruminação diferiu entre a dieta contendo $100 \%$ de concentrado e as demais. Entretanto, as médias de cada tratamento indicam que a dieta com 100\% de concentrado promoveu maior tempo de ruminação (min/ dia e min/g FDN) em comparação àquelas contendo casca de soja (Tabela 2). O tempo de ruminação em min/g FDN foi influenciado $(\mathrm{P}<0,05)$ pelo teor e pela fonte de FDN, pois foi menor para as dietas que continham casca de soja. Essa alteração no comportamento ingestivo é ocasionada pelo tamanho de partículas, uma vez que as dietas contendo 14\% de FDN apresentaram 63,5\% e $56,7 \%$ de partículas maiores que $1,18 \mathrm{~mm}$ para o bagaço de cana in natura e casca de soja, respectivamente. O mesmo foi observado para as dietas contendo $18 \%$ de FDN, com valores de $61,5 \%$ e 53,8\% de partículas maiores que 1,18 mm para bagaço in natura e casca de soja, respectivamente (Tabela 1). Segundo Mertens (1997), partículas de alimento menores que $1,18 \mathrm{~mm}$ passam pelo rúmen sem a necessidade de ruminação e esse é o tamanho mínimo para estimular a atividade de mastigação.
Diversos autores citados por Mertens (1997) demonstraram que a atividade de mastigação é uma característica que reflete as propriedades físicas e químicas dos alimentos, como a concentração de FDN, o tamanho de partículas e a umidade. Segundo Allen (1997), a fibra fisicamente efetiva é a fração do alimento que estimula a atividade de mastigação. Dependendo da fonte de FDN utilizada, o tempo de mastigação (min/dia) apresentou respostas antagônicas, de modo que, ao elevar o teor de FDN da dieta para $18 \%$, a presença da casca de soja diminuiu o tempo de mastigação $(\mathrm{P}<0,05)$; ao passo que o aumento da concentração de bagaço de cana in natura proporcionou aumento no tempo gasto com a atividade de mastigação. Os resultados da atividade de mastigação promovida pelas dietas que continham bagaço de cana in natura estão de acordo com Fimbres et al. (2002) e Rabelo et al. (2008). A superioridade das dietas contendo bagaço de cana in natura na atividade de mastigação em relação àquelas com casca de soja se deve ao fato de que a casca de soja apresenta menor efetividade de fibra em relação ao bagaço de cana in natura. Segundo Mertens (1997), em dietas com alta proporção de concentrado, a efetividade física da FDN é mais limitante para a atividade mastigatória que a substituição de FDN por carboidratos não-fibrosos.

A dieta contendo casca de soja e 18\% de FDN proporcionou menor tempo de mastigação (min/dia) em comparação à dieta controle, provavelmente porque a dieta sem fonte adicional de fibra (100C), apesar de ser composta somente por ingredientes concentrados, apresentou maior tamanho de partículas (Tabela 1). O milho utilizado foi 
grosseiramente moído, o que contribuiu para elevar a proporção de partículas maiores que $1,18 \mathrm{~mm}$, estimulando a atividade de mastigação nos animais. Além disso, a mastigação estimula a secreção de saliva e os tamponantes presentes na saliva auxiliam na neutralização dos ácidos produzidos na fermentação da matéria orgânica no rúmen. O balanço entre os ácidos produzidos e a secreção de saliva é o maior determinante do $\mathrm{pH}$ ruminal. Desse modo, o maior tempo gasto com a atividade de mastigação para os animais alimentados com a dieta contendo $100 \%$ de concentrado pode ter sido uma forma para manter o ambiente ruminal mais estável, diante de uma alimentação rica em carboidratos rapidamente fermentescíveis no rúmen e baixa proporção de alimento volumoso.

O consumo de MS, MO e FDN quando fornecidas as dietas com bagaço de cana in natura ou casca de soja foi maior $(\mathrm{P}<0,05)$ que os obtidos com a dieta com $100 \%$ de concentrado (Tabela 3). Esses resultados indicam que a presença de uma fonte adicional de fibra promove ambiente ruminal mais adequado em relação à utilização exclusiva de ingredientes concentrados, estimulando o consumo de MS. Além disso, o efeito da densidade energética da dieta é um fator que deve ser considerado, pois a dieta contendo $100 \%$ de concentrado apresenta maior densidade energética e a exigência de energia metabolizável pode ter sido atendida com menor ingestão de alimento.

Da mesma forma, Morais et al. (2007) e Araujo et al. (2008b) substituíram o feno de "coastcross" pela casca de soja na alimentação de ovinos e observaram aumento na ingestão de matéria seca à medida que a casca de soja foi incluída na dieta. Esse aumento foi atribuído à melhor digestibilidade da FDN da casca de soja e ao menor tamanho de partícula em comparação ao feno de gramínea grosseiramente picado (Morais et al., 2006).

As dietas que continham casca de soja proporcionaram maior consumo de $\mathrm{FDN}(\mathrm{P}<0,05)$ em comparação àquelas compostas por bagaço de cana in natura. Contudo, esperava-se maior consumo de FDN da dieta contendo bagaço de cana in natura e 18\% de FDN, por ela apresentar, juntamente com a dieta com 18\% de FDN e casca de soja, o maior teor de FDN. Esses resultados indicam que houve seleção dos alimentos pelos cordeiros alimentados com bagaço de cana in natura. Além disso, a menor ingestão de FDN quando fornecidas as dietas contendo bagaço de cana in natura pode estar relacionada ao maior efeito da repleção ruminal do bagaço de cana-de-açúcar, em virtude da menor digestibilidade da FDN em relação à casca de soja (Tabela 3).

A digestibiliade aparente da MS e da MO foi menor $(\mathrm{P}<0,05)$ para as dietas com bagaço de cana-de-açúcar que para aquelas com casca de soja. Da mesma forma, Araujo et al. (2008b) observaram aumento linear na digestibilidade da matéria seca à medida que substituíram feno de “coastcross" pela casca de soja, provavelmente em virtude da composição química da parede celular da casca de soja, composta basicamente por celulose e apenas 3,2\% de lignina (Silva et al., 2004), enquanto o bagaço de cana in natura possui $45,3 \%$ de celulose e $16,5 \%$ de lignina (Pires et al., 2004). Esse fato é confirmado pela diferença entre as fontes de fibra na digestibilidade da FDN.

As dietas contendo casca de soja apresentaram médias de 88,8 e $72,2 \%$ na digestibilidade aparente da MS e da

Tabela 3 - Consumo e digestibilidade aparente dos nutrientes das dietas experimentais

\begin{tabular}{|c|c|c|c|c|c|c|c|c|c|c|}
\hline \multirow[t]{3}{*}{ Item } & \multicolumn{10}{|c|}{ Dieta } \\
\hline & \multirow[t]{2}{*}{$100 C^{1}$} & \multicolumn{2}{|c|}{$\begin{array}{c}\text { Bagaço de cana-de-açúcar } \\
\text { in natura }{ }^{2}\end{array}$} & \multicolumn{2}{|c|}{ Casca de soja ${ }^{3}$} & \multirow[t]{2}{*}{$\mathrm{EPM}^{4}$} & \multicolumn{4}{|c|}{ Efeito principal $^{5}$} \\
\hline & & FDN14 & FDN18 & FDN14 & FDN18 & & Fonte & Teor & Teor $\times$ Fonte & $100 \mathrm{C} \times$ Outros \\
\hline \multicolumn{11}{|l|}{ Consumo (kg/dia) } \\
\hline Matéria seca & 0,72 & 0,94 & 0,95 & 1,11 & 0,98 & 0,20 & NS & NS & NS & 0,03 \\
\hline Matéria orgânica & 0,69 & 0,89 & 0,91 & 1,07 & 0,94 & 0,19 & NS & NS & NS & 0,02 \\
\hline $\begin{array}{l}\text { Fibra em detergente } \\
\text { neutro }\end{array}$ & 0,08 & 0,13 & 0,17 & 0,16 & 0,18 & 0,04 & 0,02 & NS & NS & $<0,01$ \\
\hline \multicolumn{11}{|c|}{ Digestibilidade aparente (\%) } \\
\hline Matéria seca & 87,2 & 82,3 & 82,6 & 88,0 & 89,6 & 1,04 & $<0,01$ & NS & NS & NS \\
\hline Matéria orgânica & 88,6 & 84,3 & 84,9 & 89,2 & 90,8 & 0,94 & $<0,01$ & NS & NS & NS \\
\hline $\begin{array}{l}\text { Fibra em detergente } \\
\text { neutro }\end{array}$ & 61,1 & 47,2 & 45,0 & 67,8 & 76,6 & 3,57 & $<0,01$ & NS & NS & NS \\
\hline
\end{tabular}

1 Dieta contendo $100 \%$ de concentrado (sem fonte adicional de FDN).

2 Dieta contendo $14 \%$ ou $18 \%$ FDN com bagaço de cana-de-açúcar in natura como fonte de FDN.

${ }^{3}$ Dieta contendo $14 \%$ ou $18 \%$ FDN com casca de soja como fonte de FDN.

4 Erro-padrão da média.

${ }^{5}$ Fonte $=$ Efeito das fontes de FDN $($ BIN $\times$ CS), Teor $=$ efeito dos teores de FDN $(14 \% \times 18 \%)$, Teor $\times$ Fonte $=$ interação entre os teores e as fontes de FDN, $100 \mathrm{C} x$ Outros = efeito das dietas com uma das fontes de FDN comparada à dieta $100 \%$ concentrado. 
FDN, respectivamente. Resultados inferiores foram obtidos por Hejazi et al. (1999), que alimentaram cordeiros com $90 \%$ de concentrado e $10 \%$ de casca de soja como única fonte de fibra e obtiveram digestibilidade da matéria seca e da FDN de 80,4 e 46,8\%, respectivamente. Por outro lado, avaliando a digestibilidade in vitro da casca de soja, Silva et al. (2004) obtiveram valores de 76,9 e 85,6\% para DIVMS e DIVFDN, respectivamente. Segundo esses autores, a maior concentração de proteína bruta e baixo teor de lignina são fatores que favorecem a digestibilidade desse ingrediente.

Os resultados obtidos para digestibilidade aparente da MS $(87,2 \%)$ e da FDN $(61,1 \%)$ com a dieta sem fonte adicional de fibra (100C) pode ser atribuído à característica física do milho utilizado. Segundo Vance et al. (1972), o milho pouco processado (grão inteiro ou moído grosseiramente) exerce efeito de forragem no rúmen em comparação ao milho moído fino. A proporção de partículas maiores que $1,18 \mathrm{~mm}$ da dieta contendo $100 \%$ de concentrado (Tabela 1) teria sido menor se o milho utilizado neste trabalho fosse finamente moído. O milho utilizado aumentou a proporção de partículas maiores que $1,18 \mathrm{~mm}$, estimulando a atividade de mastigação nos animais e, possivelmente, a produção de saliva (Allen, 1997), criando ambiente ruminal mais estável para a fermentação microbiana e favorecendo a digestibilidade da MS, MO e FDN.

\section{Conclusões}

A utilização da casca de soja como fonte de fibra proporciona menor atividade de mastigação e maior digestibilidade dos nutrientes que o uso do bagaço de cana-de-açúcar in natura. A casca de soja pode ser utilizada como única fonte adicional de fibra em detergente neutro em dietas contendo alto teor de concentrado na alimentação de cordeiros.

\section{Referências}

ALLEN, M.S. Relationship between fermentation and acid production in the rumen and requirement for physically effective fiber. Journal of Dairy Science, v.80, p.1447-1462, 1997.

ANDERSON, K.N.; MERRIL, J.K.; MCDONNELL, M.L. et al. Digestibility and utilization of mechanically processed soybean hulls by lambs and steers. Journal of Dairy Science, v.66, n.11, p.2965-2975, 1988.

ARAUJO, R.C.; PIRES, A.V.; SUSIN, I. et al. Milk yield, milk composition, eating behavior, and lamb performance of ewes fed diets containing soybean hulls replacing coastcross (Cynodon species) hay. Journal of Animal Science, v.86, p.3511-3521, 2008a.

ARAUJO, R.C.; PIRES, A.V.; SUSIN, I. et al. Apparent digestibility of diets with combinations of soybean hulls and coastcross
(Cynodon sp) hay offered to ram lambs. Scientia Agricola, v.65, p.581-588, 2008b.

ARMENTANO, L.; PEREIRA, M. Measuring the effectiveness of fiber by animal response trials. Journal of Dairy Science, v.80, p.1416-1425, 1997.

ASSOCIATION OF OFFICIAL ANALYTICAL CHEMISTS AOAC. Official methods of analysis. 15.ed. Arlington, 1990. 1117p.

BULLE, M.L.M.; RIBEIRO, F.G.; LEME, P.R. et al. Desempenho de tourinhos cruzados em rações de alto teor de concentrado com bagaço de cana-de-açúcar como único volumoso. Revista Brasileira de Zootecnia, v.31, n.1, p.444-450, 2002.

FIMBRES, H.; KAWAS, J.R.; HERNÁNDEZ-VIDAL, G. et al. Nutrient intake, digestibility, mastication and ruminal fermentation of lambs fed finishing ration with various forage levels. Small Ruminant Research, v.43, p.275-281, 2002 .

GOERING, H.K.; VAN SOEST, P.J. Forage fiber analysis (apparatus, reagents, procedures and some applications). Washington D.C.: Agricultural Research Service, 1970. $19 p$.

HEJAZI, S.; FLUHARTY, F.L.; PERLEY, J.E. et al. Effects of corn processing and dietary fiber source on feedlot performance, visceral organ weight, diet digestibility, and nitrogen metabolism in lambs. Journal of Animal Science, v.77, p.507-515, 1999.

HENRIQUE, W.; BELTRAME, J.A.; LEME, P.R. et al. Avaliação da silagem de grãos de milho úmido com diferentes volumosos para tourinhos em terminação: Desempenho e características de carcaça. Revista Brasileira de Zootecnia, v.36, n.1, p.183-190, 2007.

IPHARRAGUERRE, I.R.; CLARK, J.H. Soyhulls as an alternative feed for lactating dairy cows: a review. Journal of Dairy Science, v.86, p.1052-1073, 2003.

MERTENS, D.R. Creating a system for meeting the fiber requirements of dairy cows. Journal of Dairy Science, v.80, p.1463-1481, 1997.

MORAIS, J.B.; SUSIN, I.; PIRES, A.V. et al. Comportamento ingestivo de ovinos e digestibilidade aparente dos nutrientes de rações contendo casca de soja. Pesquisa Agropecuária Brasileira, v.41, n.7, p.1157-1164, 2006.

MORAIS, J.B.; SUSIN, I.; PIRES, A.V. et al. Substituição do feno de "coastcross" (Cynodon sp.) por casca de soja na alimentação de borregas confinadas. Ciência Rural, v.37, n.4, p.1073- 1078, 2007.

NATIONAL RESEARCH COUNCIL - NRC. Nutrient requirements of sheep. 6.ed. Washington, D.C.: National Academy Press, 1985. 99p.

PIRES, A.J.V.; REIS, R.A.; CARVALHO, G.G.P. et al. Degradabilidade do bagaço de cana-de-açúcar tratado com amônia anidra e, ou, sulfeto de sódio. Revista Brasileira de Zootecnia, v.33, p.1071-1077, 2004.

RABELO, M.M.A.; PIRES, A.V.; SUSIN, I. et al. Avaliação do efeito do bagaço de cana-de-açúcar in natura obtido por dois métodos sobre o desempenho e o comportamento ingestivo de bovinos de corte. Arquivo Brasileiro de Medicina Veterinária e Zootecnia, v.60, p.698-704, 2008.

SARWAR, M.; FIRKINS, J.L.; EASTRIDGE, M.L. Effect of replacing neutral detergent fiber of forage with soyhulls and corn gluten feed for dairy heifers. Journal of Dairy Science, v.74, p.1006-1017, 1991.

SILVA, D.C.; KAZAMA, R.; FAUSTINO, J.O. et al. Digestibilidade in vitro e degradabilidade in situ da casca de grão de soja, resíduo de soja e casca de algodão. Acta Scientiarum Animal Science, v.26, n.4, p.501-506, 2004.

STATISTICAL ANALYSIS SYSTEM - SAS. User's guide: statistic. 6.ed. Cary, 1999. 956p.

TURINO, V.F.; SUSIN, I; PIRES, A.V. et al. Casca de soja na alimentação de cordeiros confinados: desempenho e 
características da carcaça. Ciência Animal Brasileira, v.8, n.3, p.405-503, 2007.

VANCE, R.D.; PRESTON R.L.; KLOSTERMAN, E.W. et al. Utilization of whole shelled and crimped corn grain with varying proportions of corn silage by growing finishing steers. Journal of Animal Science, v.35, p.598-605, 1972.
VAN SOEST, P.J.; ROBERTSON, J.B.; LEWIS, B.A. Methods for dietary fiber, neutral detergent fiber, and nonstarch polysaccharides in relation to animal nutrition. Journal of Dairy Science, v.74, p.3583-3597, 1991.

1WEIDNER, S.J.; GRANT, R.J. Soyhulls as a replacement for forage fiber in diets for lactating dairy cows. Journal of Dairy Science, v.77, p.513-521, 1994. 